\title{
Effect of the Hydrate Saturation of the Pores of Coal Bed on the Gradient of Gas Pressure and the Rise of Outburst Danger
}

\author{
Vyacheslav Smirnov ${ }^{1}$, Valery Dyrdin ${ }^{1,}$, Tatyana $\mathrm{Kim}^{1}$, and Andrey Manakov ${ }^{2}$ \\ ${ }^{1}$ T.F. Gorbachev Kuzbass State Technical University, 650000, Kemerovo, Russian Federation \\ ${ }^{2}$ A.V. Nikolaev Institute of Inorganic Chemistry, SBRAS, 630090, Novosibirsk, Russian Federation
}

\begin{abstract}
A substantial fraction of methane in undisturbed coal beds is present in the condensed latent state, so that methane evolution from coal may be not always quantitatively predicted reliably. On the basis of experimental data, an equation expressing the amount of gas hydrate through the sorption capacity and actual humidity of coal is obtained. Analysis showed that the gradient of gas pressure in the marginal zone of a coal bed is linearly dependent on the saturation of the pore space with the hydrate. The high gradient of gas pressure and high gas content of coal beds along with local disruption of coal and re-distribution of rock pressure are the major factors causing instantaneous outbursts of coal and gas.
\end{abstract}

\section{Introduction}

Sudden outburst of coal and gas is a complex multifactorial process, and its description within the known physical models is rather complicated [1-4]. The existing approaches to the prediction and prevention of the sudden outbursts of coal and gas put forward the problem to reveal the major factors that accompany the outburst or, quite contrary, hinder it [5-8]. To take into account the integrated action of the formation pressure, elastic deformations of the bed, gas content etc., each of the parameters is expressed through the forces accompanying or preventing the outburst or through the accumulated elastic energy and the work necessary for the destruction of coal and its transport into the mine [8-11]. The major contribution into the active forces accompanying a sudden outburst of coal and gas is made by the gradient of rock and gas pressure in the marginal zone of the bed. The passive forces are determined by the limiting friction forces acting at the perimeter of the bottomhole formation zone and the coefficient of adhesion. The potential energy realized during a sudden outburst of coal and gas is formed in the elastic deformations of coal bed and host rocks, as well as in coal methane which is under high pressure. The total amount of gas present in coal and the rate of initial gas evolution determine the danger of outburst from the marginal zone, so it becomes urgent to study the forms connecting coal methane with the solid substance of coal [8-9, 12-13].

\footnotetext{
*Corresponding author: dvv.fiz@,kuzstu.ru
} 


\section{Materials and Methods}

Gas hydrates are one of the possible forms in which methane is present in coal beds [1215]. Up to 160 volumes of gas (reduced to the normal conditions) are released from one volume of the hydrate. For the coal beds in Kuzbass, the pressure of gas and rocks, as well as bed temperature coincide in many cases with the thermobaric boundary of gas hydrate stability. Mining causes disturbance of coal bed pressure and decomposition of methane hydrate. It is quite probable that the high rate of initial gas evolution, which is used by many researchers as an indicator of coal bed outburst danger, is connected with the decomposition of methane hydrate or other compounds that were formed previously in the coal bed. If the formation pressure is higher than the curve of gas hydrate equilibrium, methane is present in the condensed phase and it is difficult to detect it and measure its amount.

In the present work we discuss the results of experiments on the measurement of the amount of gas hydrate in natural coal with different degree of metamorphism, and estimate the effect of gas evolved during the decomposition of the gas hydrate on the gradient of gas pressure in the marginal zone of the coal bed.

\section{Results and discussion}

The conditions for the formation of gas hydrate in coal beds are achieved in the case if the sufficient amounts of gas and water are present, coal bed temperature is about $+5 \ldots+15^{\circ} \mathrm{C}$, and the pressure is within the range $2 \ldots 12 \mathrm{MPa}$. Water and gas are always present in some amounts in coal beds. Pressure and temperature values that are equilibrium for the hydrate are determined in particular by the composition of the hydrate-forming gas, and in many cases they are close to the conditions of real coal beds. As an example [13], it is to be indicated that at a temperature of $+7^{\circ} \mathrm{C}$, the pressure necessary for the stability of methane hydrate is $5.3 \mathrm{MPa}$, while the pressure necessary for the stability of the hydrate of carbon dioxide is 3.0 MPa. The hydrate of natural gas is stable at this temperature, even if the pressure decreases to $2.2 \mathrm{MPa}$. In our experiments, natural gas contained $89 \%$ methane, $6 \%$ nitrogen, $3 \%$ oxygen, $1.2 \%$ carbon dioxide and other gases. Coal is a porous continuous medium in which substantial parts of methane and water are bound with the solid matter of coal. In this connection, is it necessary to measure the hydrate capacity, that is, the maximal amount of the hydrate embedded in natural coal of one or another rank. The maximal amount of the hydrate is determined by the volume of coal pores with gas and water, sorbed by coal [14-18].

We carried out experiments with a series of coal samples in which we measured the amount of methane evolved during the decomposition of gas hydrates formed in these samples at a temperature and pressure close to the temperature and pressure in real coal beds $[12,13,17,18]$. Experiments were carried out in a closed autoclave, the amount of evolved gas was calculated from pressure rise. Hydrate decomposition was initiated by slow temperature rise within the range $0^{\circ} \mathrm{C} \ldots+15^{\circ} \mathrm{C}$. Experiments with the samples of coal with different degrees of metamorphism demonstrated that each coal sample is characterized by a definite threshold of humidity below which no hydrate is formed in coal; when this threshold is exceeded, only a part of water sorbed above the threshold of hydrate formation is involved in the formation of the hydrate. To calculate the amount of hydrate, A and $\mathrm{B}$ constants were determined for each coal sample: 
$A=\frac{W_{t r h}}{W_{91}}-$ the ratio of humidity corresponding to the threshold of hydrate formation $W_{t r h}$, to coal humidity reached in the atmosphere with $91 \%$ relative humidity $W_{91}$;

$$
B=\frac{\Delta w_{h}}{\Delta w} \text { - the ratio of the amount of water additionally involved into hydrate }
$$

formation to an increase in coal humidity above the threshold of hydrate formation.

The values of A and B coefficients for coal samples studied by us are shown in Table 1, along with the calculated amount of water $\left(w_{h}\right)$ and gas $\left(V_{h}, \mathrm{~m}^{3} / \mathrm{t}\right)$, which is present in the form of hydrate at the humidity reached in the atmosphere with $91 \%$ relative humidity.

The amount of hydrate, and therefore the volume of gas $\left(V_{h}\right)$ involved in the formation of the hydrate may be estimated from the actual humidity of coal under natural conditions $(w)$ using the equation:

$$
V_{h}=2.1 \cdot\left(w-A \cdot W_{91}\right) B
$$

Equation (1) determines the volume of gas forming the hydrate from the internal moisture. Free (external) moisture is present in the cracks and large pores; the regularities of hydrate formation from this water obey the laws of hydrate formation from bulk water.

Gas hydrate is formed in a coal bed if the pressure and temperature correspond to the conditions of hydrate stability. The data shown in Table 1, together with equation (1), allow calculating the amount of hydrate formed in specific coal samples. The constants A and B change monotonously for the entire sequence of coal metamorphism, so these values may be spread to other coal samples with similar properties measuring only the actual coal humidity and the amount of water sorbed by coal in the atmosphere with the relative humidity of $91 \%$.

Table 1. Coefficients of equation (1) for the samples of coal of several grades, the volume of gas

\begin{tabular}{|c|c|c|c|c|c|c|}
\hline Coal & $W_{91}, \%$ & B & $\mathbf{A}$ & $W_{\text {trh }}, \%$ & $\mathrm{wh}_{\mathrm{h}}, \%$ & $\mathbf{V}, \mathbf{m}^{3} / \mathbf{t}$ \\
\hline D & 9.0 & 0.51 & 0.83 & 7.5 & 0.8 & 1.6 \\
\hline G & 10.2 & 0.43 & 0.67 & 6.8 & 1.4 & 3.0 \\
\hline G & 5.8 & 0.48 & 0.66 & 3.8 & 1.0 & 2.0 \\
\hline GZh & 5.0 & 0.59 & 0.73 & 3.7 & 0.8 & 1.7 \\
\hline $\mathrm{Zh}$ & 3.4 & 0.64 & 0.58 & 2.0 & 0.9 & 1.9 \\
\hline K & 2.4 & 0.64 & 0.44 & 1.0 & 0.8 & 1.8 \\
\hline $\mathrm{T}$ & 3.3 & 0.63 & 0.53 & 1.8 & 1.0 & 2.1 \\
\hline A & 4.0 & 0.57 & 0.39 & 1.6 & 1.4 & 2.9 \\
\hline
\end{tabular}
involved in the formation of gas hydrate.

It was also established as a result of experimental studies that the decomposition of gas hydrate occurs immediately after the violation of the thermobaric conditions of gas hydrate stability. In all experiments, the decomposition of the gas hydrate was limited by the rate of the changes of external parameters. The amount of gas evolved during hydrate decomposition is comparable with the amount of gas which is present in the sorbed state. In the case of the disturbance of equilibrium in the coal bed, these amounts of gas are summed thus increasing the volume of gas evolved in the marginal zone, which causes an increase in 
the gradient of gas pressure and the formation of the situation fraught with the danger of outburst $[5,8,9]$.

Below we will estimate the effect of hydrate saturation of a coal bed with high gas content on the state of the bottomhole formation zone. When the outcrop surface is created, the gas starts to filter out from deeper regions to the surface of the coalface $z=0$, at which the pressure is equal to atmospheric pressure $P_{0}$. The flow is considered to be isothermal $T=$ const , the section of the channels through which the gas is filtered is considered to be constant, as well as filtration coefficient $k$ and the coefficient of dynamic viscosity $\mu$. The following designations will be introduced:

$\mathrm{Oz}, \mathrm{z}$ - the axis directed to the depth of the bed along the coalface axis, coordinate;

$w(z), w-$ the current gas flow rate (averaged over the cross section);

$\rho(z)=\frac{M}{R T} P(z), \rho-$ gas density varying along the $O z$ axis, depending on gas pressure - $P(z), P$ then $M, R$ - the molar mass of the gas, the universal gas constant; $G$ specific mass consumption of the gas in the flow. The area of the coal bed through which filtration proceeds is accepted to be equal to unity.

At the first stage, we accept the absence of gas sources; in this case the condition of steady flow is equivalent to the constant gas consumption:

$$
\rho w=-G, \text { or } P w=\frac{-G R T}{M} .
$$

Minus arises because the gas flow is directed against the chosen axis $O z$. Darcy law determining the relations between the rate of filtration flow and the distribution of gas pressure is:

$$
w=-\frac{k}{\mu} \frac{\partial P}{\partial z}
$$

Substituting (3) into equation (2) we obtain:

$$
P \frac{\partial P}{\partial z}=\frac{G R T \mu}{M k},
$$

The approximation of the steady flow with constant consumption is equivalent to the requirement that the pressure at the coalface edge is always constant and equal to $P_{0}$, while at a distance of $L$ from the coalface pressure is also constant and equal to $P_{1}$. The constant gas flow through any cross section of the edge zone and the constant gas pressure are provided by the gradual decomposition of gas hydrate. The gradient of gas pressure may be expressed from equation (4):

$$
\frac{\partial P}{\partial z}=\frac{P_{1}^{2}-P_{0}^{2}}{L \cdot P(z)}
$$

The mass gas flow rate corresponding to this formulation of the problem is equal to

$$
G=\frac{\left(P_{1}^{2}-P_{0}^{2}\right) M k}{L \cdot 2 R T \mu} \text {. }
$$


The largest gradient of gas pressure (6) is achieved near the coalface when $P(z)=P_{0}$.

$$
\frac{\partial P}{\partial z}=\frac{P_{1}^{2}-P_{0}^{2}}{L \cdot P_{0}}
$$

The gradient of gas pressure is a criterion for the occurrence of sudden outbursts of coal and gas. Pressure $P_{l}$ may be determined quite reliably, while the distance $L$ from the coalface to the depth of the massif on which the pressure is maintained is a variable parameter depending on the nature of strain distribution and the coefficient of permeability, therefore, finally it depends on the gas flow value. This distance increases gradually with an increase in time because of the consumption of gas present in the sorbed state and in the form of gas hydrate. In the case of the uniform velocity of coalface movement, methane desorption from coal occurs within the whole edge zone increasing the filtration flux and the gradient of gas pressure in the edge zone of the bed. Within the framework of our model, we will accept that sorbed methane in the amount of $n_{0}$ is evolved at a distance of $L$ from the coalface front. In the same point, gas evolved during the decomposition of gas hydrates enters of coal massif. If hydrate saturation $\beta$ and the amount of desorbing gas $n_{0}$ are estimated through the mass of the gas evolved during hydrate decomposition and during desorption in a unit volume of coal, the mass flow rate characterizing the filtration flow may be expressed through the displacement of the front at which pressure is equal to $P_{l}$.

$$
G=\left(\beta+n_{0}\right) \frac{d L}{d t}
$$

The following equation is obtained from (7) and (9):

$$
L \frac{d L}{d t}=\frac{\left(P_{1}^{2}-P_{0}^{2}\right) M k}{2 R T \mu\left(\beta+n_{0}\right)}
$$

The solution of this equation may be written using several methods:

$$
\begin{aligned}
& L=\sqrt{\frac{\left(P_{1}^{2}-P_{0}^{2}\right) M k}{2 R T \mu\left(\beta+n_{0}\right)} \sqrt{t}} \\
& \frac{d L}{d t}=\sqrt{\frac{\left(P_{1}^{2}-P_{0}^{2}\right) M k}{8 R T \mu\left(\beta+n_{0}\right) \cdot t}}=\frac{L}{2 t} .
\end{aligned}
$$

The distance $L$ (11) to the front of constant pressure increases with time, but the rate at which this distance increases falls down (12), and gas flow (9) through the edge zone of the coal face decreases. On the other hand, the displacement of coalface front causes a decrease in $L$, so a steady $L$ value is achieved for the constant rate of coalface movement, for which the velocity of high pressure front is equal to the velocity of coalface movement. Directly from (10), it is possible to write an expression for the rate of the change of this length and to equate it with the average velocity $(u)$ of the movement of coalface front, to obtain the right-hand part of equation (8): 


$$
\frac{P_{1}^{2}-P_{0}^{2}}{P_{0} L}=\frac{2 \mu}{\rho_{0} k}\left(\beta+n_{0}\right) u .
$$

In the latter expression $\rho_{0}=\frac{P_{0} M}{R T}$ is gas density in the filtration flow near the coalface front. The gradient of gas pressure in the edge zone of coal bed may be expressed through hydrate saturation and the average velocity of coalface movement:

$$
\frac{\partial P}{\partial z}=\frac{2 \mu}{\rho_{0} k}\left(\beta+n_{0}\right) u \text {. }
$$

Thus, the gradient of gas pressure is proportional to the product of the velocity of coalface movement and the sum of the amounts of desorbed gas and the gas evolved during the decomposition of gas hydrate. The gradient of gas pressure determines the active forces transforming coal destroyed by the forces of formation pressure into the suspended state and transporting it along the mine.

\section{Conclusions}

1. It is established that coal methane is able to pass into the condensed state in natural coal and to form gas hydrate. The amount of gas hydrate formed in coal bed is expressed through the humidity and sorption capacity of coal.

2. It is proved that hydrate decomposition increases the gas balance of a coal bed, leads to an increase in the gradient of gas pressure and filtration flux in the edge zone - one of the major factors affecting the formation of situations with the danger of outburst.

\section{References}

1. A. Fisne, O. Elsen, Nat Hazards, 74, 1363 (2014)

2. Sh. Li, T. Zhang, Mining Science and Technology, 20, 0209 (2010)

3. Qi. Tu, Yu. Cheng, P. Guo, Ji. Jiang, L. Wang, R. Zhang, Rock Mech. Eng., 49, 3769 (2016)

4. Yu. Cao, D. He, D. C. Glick, International Journal of Coal Geology, 48, 47 (2001)

5. W. U. Dongmei, Zh. Yuemin, Ch. Yuanping, A. N. Fenghua, Mining Science and Technology, 20, 0723 (2010)

6. F. Hao, M. Liu, L. Sun, Procedia Engineering, 26, 699 (2011)

7. S. Jacek, Fuel, 115, 288 (2014)

8. A. D. Alekseev, E. P. Feldman, Tech Phys Lett., 34, 609 (2008)

9. E. P. Fel'dman, T. A. Vasilenko, and N. A. Kalugina, Journal of Mining Science, 50, 448 (2014)

10. A. V. Shadrin, Journal of Mining Science, 52, 670 (2016)

11. V. N. Oparin, T. A. Kiryaeva, V. Yu. Gavrilov, R. A. Shutilov, A. P. Kovchavtsev, A. S. Tanaino, V. P. Efimov, I. E. Astrakhantsev, I. V. Grenev, Journal of Mining Science, 50, 191 (2014)

12. V. G. Smirnov, V. V. Dyrdin, A. Yu. Manakov, Z. R. Ismagilov, T. P. Adamova, Chemistry for Sustainable Development, 24, 499 (2016) 
13. V. V. Dyrdin, V. G. Smirnov, T. L. Kim, A. Yu. Manakov, A. A. Fofanov, I. S. Kartopolova, Russian Physics Journal, 60, 206 (2017)

14. A. M. Bustin, R. M. Bustin, I. L. Moudrakovski, S. Takeya, J. A. Ripmeester, Energy Fuels, 30, 88 (2016)

15. D.-L. Zhong, K. Ding, Yi-Yu Lu, Jin Yan, W.-L. Zhao, Applied Energy, 162, 1619 (2016)

16. Yi. Cai, D. Liu, Zh. Pan, Ya. Yao, Ju. Li, Yo. Qiu, Fuel 103, 258 (2013)

17. V. G. Smirnov, V. V. Dyrdin, Z. R. Ismagilov, A. Yu. Manakov, E. A. Ukraintseva, G. V. Villevald， T. D. Karpova， I. S. Terekhova， A. G. Ogienko， S. Yu. Lyrshchikov, Fuel, 166, 188 (2016)

18. V. G. Smirnov, A. Yu. Manakov, V. V. Dyrdin, Z. R. Ismagilov, E. S. Mikhailova, T. V. Rodionova, G. V. Villevald, V. Yu. Malysheva, Fuel, 228, 123 (2018) 